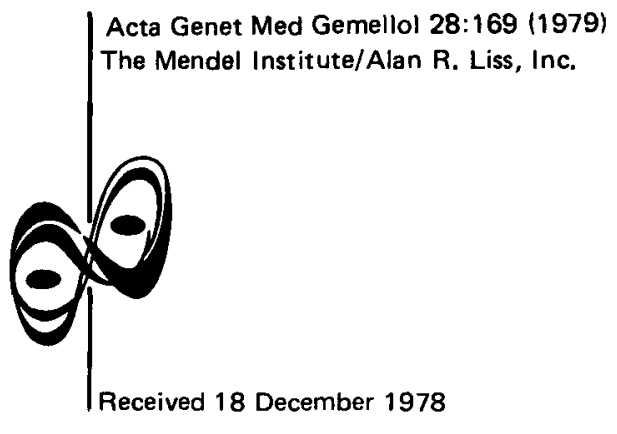

$\overline{\text { Letters to the Editor }}$

\title{
Applicability of Selvin's Efficiency Measure in Twin Zygosity Diagnosis by Genetic Systems
}

\author{
Seppo Sarna and Jaakko Kaprio \\ Department of Public Health Science, University of Helsinki
}

In regard to the correspondence between Drs. Selvin and Chakraborty $[1,4]$ we would like to comment briefly. Dr. Selvin's reply to Chakraborty [4] clarifies and stresses the restrictive assumption on which his results are valid, but it is hard to believe that he really meant to restrict the applicability of his efficiency measure only to the case of genotypes, because when he discusses his results he clearly refers to the practical applicability of his measure [3].

As a theoretical consideration Dr. Selvin's note is correct, but since in practice we must operate with phenotypic information, the efficiency measure in the form proposed is not very valuable. If the population gene frequencies are known, generalized phenotypic consideration can be presented as shown by us [2]. The relative efficiencies of the markers used by Dr. Selvin change when phenotypes are considered; for example, the relative efficiency of the Duffy system will worsen compared to the polyallelic systems.

\section{REFERENCES}

1. Chakraborty R (1979): Twin zygosity diagnosis by genetic systems: An efficiency evaluation. A comment to a paper by Selvin (1977): Acta Genet Med Gemellol 28:77-79.

2. Sama S, Kaprio J (1979): Dependency of concordance probability on gene frequencies in genetic systems for the diagnosis of twin zygosity. Acta Genet Med Gemellol 28:133-138.

3. Selvin (1977): Efficiency of genetic systems for diagnosis of twin zygosity. Acta Genet Med Gemellol $26: 81-82$.

4. Selvin S (1979): A reply to Chakraborty. Acta Genet Med Gemellol 28:80. 